\title{
First total synthesis of Boehmenan
}

\author{
YAMU XIA ${ }^{\mathrm{a}, *}, \mathrm{XIAOLI}^{\mathrm{DAI}}{ }^{\mathrm{a}, *}, \mathrm{HAIXIN}$ LIU $^{\mathrm{a}}$ and CHEN CHAI ${ }^{\mathrm{b}}$ \\ ${ }^{a}$ College of Chemical Engineering, Qingdao University of Science and Technology, Qingdao 266042, \\ People's Republic of China \\ ${ }^{\mathrm{b}}$ The first Affiliated Hospital of Lanzhou University, Gansu 730000, People's Republic of China \\ e-mail: xiaym@qust.edu.cn; dxlqust@163.com
}

MS received 27 April 2013; revised 29 July 2013; accepted 14 November 2013

\begin{abstract}
The first total synthesis of dilignan Boehmenan has been achieved. A biomimetic oxidative coupling of the ferulic acid methyl ester in the presence of silver oxide is the crucial step in the synthesis sequence, generating the dihydrobenzofuran skeleton. Hydroxyl group was protected with DHP and reducted with $\mathrm{LiAlH}_{4}$ to afford the intermediate diol. The diol was condensated with the derivative of ferulic acid, then removed the protecting groups, to get Boehmenan. Meanwhile, a study on the ring-opening reaction of the intermediate dihydrobenzofuran neolignan under base conditions was described.
\end{abstract}

Keywords. Boehmenan; dihydrobenzofuran neolignan; dilignan; biomimetic oxidative coupling; ring-opening reaction mechanism.

\section{Introduction}

The structurally novel lignan Boehmenan (1) was first isolated in 2001 by Seca et al. from the bark of Kenaf (Hibiscus cannabinus), which is an annual dicotyledonous herbaceous plant and well-known in Asia and Africa. ${ }^{1}$ In 2005, Wu et al. reported that Boehmenan was isolated from the stems of Hibiscus taiwanensis, co-occurring with a structurally diverse set of natural products. ${ }^{2}$ Rudiyansyah et al. isolated it together with another nine compounds by the phytochemical exploration of a wood bark extract from Durio zibethinus in $2006 .{ }^{3}$ Cytotoxicity-guided fractionation of the stems of Helicteres hirsuta, led to the isolation and identification of Boehmenan by Chin et al. in the same year. ${ }^{4}$ In addition, Sasaki et al. extracted it from the whole plants of Sambucus adnata and reported the evaluation of the PTP1B inhibitory activities of it in 2011. The kinetic analysis indicated that Boehmenan in hibits PTP1B activity in a competitive manner. ${ }^{5}$

Boehmenan, with a dihydrobenzofuran skeleton, is formed by polymerization of four phenylpropanoid units. This compound belongs to the very interesting class of dilignan on account of their great number of structural possibilities. Dilignan family are found in all part of plants ${ }^{6,7}$ and display biological activities including antioxidant ${ }^{8}$ and antituberculosis activities ${ }^{9}$ and inhibitory effects on the growth of dicotyledons. ${ }^{10}$ Although many dilignans with broad

\footnotetext{
*For correspondence
}

application prospect have been found in nature, only a few have been synthesized. In 2010, our team developed a novel synthetic route of dilignan threo- $( \pm)$ diferuloysecoisolariciresinol. The method involved two Stobbe reactions to construct the skeleton of lignan, and then condensed with ferulaic acid to give threo- $( \pm)$ diferuloysecoisolariciresinol. ${ }^{11}$

Here, we report the first total synthesis of another dilignan Boehmenan. The synthesis was based on a strategy involving biomimetic oxidative coupling to give the key intermediate dihydrobenzofuran neolignan, and then treated with derivative of ferulic acid to obtain the natural product Boehmenan as shown in scheme 1. Furthermore, the reaction conditions of biomimetic oxidative coupling are described, and the possible ring-opening reaction mechanisms of dihydrobenzofuran compound are discussed in this paper.

\section{Experimental}

\subsection{Materials and apparatus}

The reagents and the solvents used in this study were of analytical grade and were used without further purification. Melting points were determined in open capillaries and are uncorrected. The purity of the compounds was checked by TLC on silica gel GF254 plates using UV/Iodine as visualizing agent and silica gel (200-300 mesh) was used for column chromatographic purification. The ${ }^{1} \mathrm{HNMR}$ and ${ }^{13} \mathrm{CNMR}$ spectra were recorded 


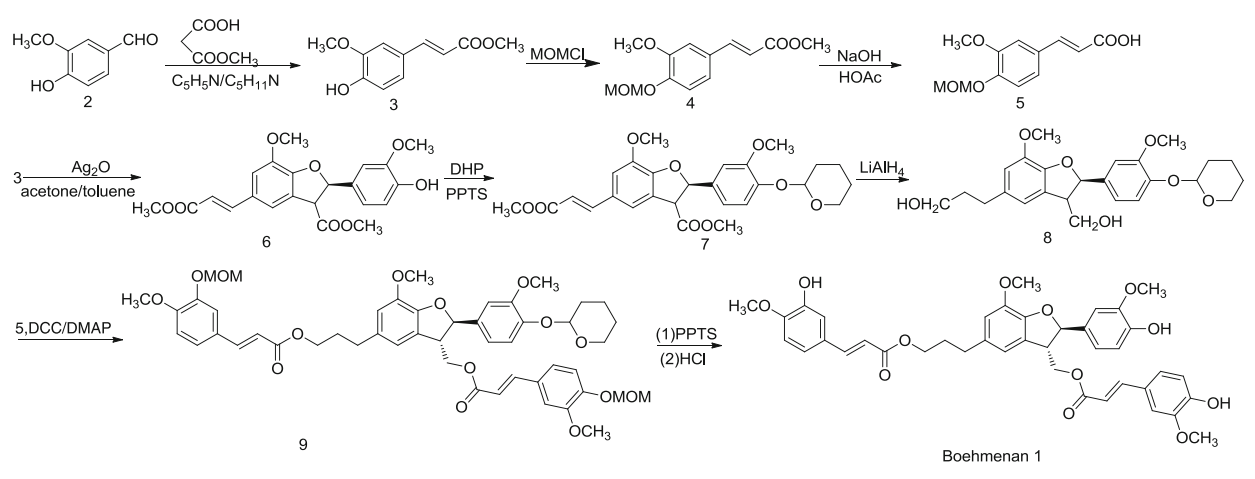

Scheme 1. Synthesis of Boehmenan.

on a Brucker $A M-500 \mathrm{MHz}$ spectrometers. HRMS were obtained on a Bruker Daltonics APEXII47e spectrometer.

\subsection{Synthesis of (E)-ferulic acid methyl ester (3)}

A mixture of vanillin $(8.58 \mathrm{~g}, 56.4 \mathrm{mmol})$, methyl hydrogen malonate $(13.32 \mathrm{~g}, 112.8 \mathrm{mmol})$ and piperidine $(0.72 \mathrm{~g}, 8.5 \mathrm{mmol})$ in pyridine $(13 \mathrm{~mL})$ were heated at $100^{\circ} \mathrm{C}$ for $4 \mathrm{~h}$. The residue was poured into diluted hydrochloric acid solution which was cooled at $0^{\circ} \mathrm{C}$. After three days, the crude product obtained was filtered and recrystallized from ethanol to give compound 3 (10.40 g).

2.2a (E)- ferulic acid methyl ester (3): This compound was obtained as a white solid, mp: $63-64^{\circ} \mathrm{C}$; $89 \%$ yield; HRMS calcd for $\mathrm{C}_{11} \mathrm{H}_{12} \mathrm{O}_{4} 208.0737$, found 208.0751; ${ }^{1} \mathrm{H}$ NMR $\left(500 \mathrm{MHz}, \mathrm{CDCl}_{3}\right) \delta 3.69$ and $3.91\left(\mathrm{~s}, 6 \mathrm{H}, 2 \times \mathrm{OCH}_{3}\right), 6.39(\mathrm{~d}, 1 \mathrm{H}, J=16.0 \mathrm{~Hz}$, $\mathrm{ArCH}=\mathrm{CH}), 6.87-7.30(\mathrm{~m}, 3 \mathrm{H}, \mathrm{ArH}), 7.59(\mathrm{~d}, 1 \mathrm{H}, J$ $=16.0 \mathrm{~Hz}, \mathrm{ArCH}=\mathrm{CH}), 8.11(\mathrm{~s}, 1 \mathrm{H}, \mathrm{OH}) ;{ }^{13} \mathrm{C} \mathrm{NMR}$ $\left(125 \mathrm{MHz}, \mathrm{CDCl}_{3}\right) \delta 51.49,56.36,111.42,115.55$, $116.06,123.75,127.42,145.66,148.67,149.98,167.87$ $(\mathrm{C}=\mathrm{O})$. The data are consistant with the literature. ${ }^{12}$

\subsection{Synthesis of methyl (E)-4-methoxymethyl-3- methoxycinnamate $(4)^{13,14}$}

2.3a The preparation of $\mathrm{MOMCl}$ : In a $1 \mathrm{~L}$ roundbottomed flask fitted with a stopper carrying a reflux condenser and a glass tube reaching nearly to the bottom of the flask are placed methyl alcohol (100.00 g, $3.1 \mathrm{~mol}$ ) and formaldehyde (72.00 g, $2.4 \mathrm{~mol})$. A rapid stream of hydrogen chloride is run into the mixture, which is cooled with running water. In about two hours a layer of chloromethyl ether begins to appear. The stream of hydrogen chloride is continued for two or three hours longer until the solution is saturated. The layer of chloromethyl ether is then separated. The water layer is saturated with calcium chloride, and more ether separates. This is added to the main portion, which is then dried over calcium chloride and fractionally distilled. The yield of $\mathrm{MOMCl}$ boiling at $55-60^{\circ} \mathrm{C}$ is about $150 \mathrm{~g}$.

$2.3 \mathrm{~b}$ The synthesis of methyl (E)-4-methoxymethyl3-methoxycinnamate (4): A mixture of compound 3 $(2.81 \mathrm{~g}, 13.5 \mathrm{mmol})$, potassium carbonate $(5.59 \mathrm{~g}$, $40.5 \mathrm{mmol})$ in acetone $(20 \mathrm{~mL})$, was stirred for $1 \mathrm{~h}$ at room temperature. Then, $\mathrm{MOMCl}(2.18 \mathrm{~g}, 27.1 \mathrm{mmol})$ was added drop-wise. Stirring was continued for $3 \mathrm{~h}$ and the reaction mixture was then quenched with aqueous ammonium chloride. The mixture was extracted with ethyl acetate, dried over $\mathrm{MgSO}_{4}$, concentrated under vacuum to give 4 (3.15 g).

\section{3c Methyl(E)-4-methoxymethyl-3-methoxycinnamate}

(4): This compound was obtained as a colourless oil, 93\% yield; HRMS calcd for $\mathrm{C}_{13} \mathrm{H}_{16} \mathrm{O}_{5} 252.0998$, found 252.1004; ${ }^{1} \mathrm{H} \mathrm{NMR}\left(500 \mathrm{MHz}, \mathrm{CDCl}_{3}\right) \quad \delta 3.46(\mathrm{~s}, 3 \mathrm{H}$, $\left.\mathrm{OCH}_{3}\right), 3.70\left(\mathrm{~s}, 3 \mathrm{H}, \mathrm{OCH}_{3}\right), 3.82\left(\mathrm{~s}, 3 \mathrm{H}, \mathrm{OCH}_{3}\right), 5.23$ $\left(\mathrm{s}, 2 \mathrm{H}, \mathrm{OCH}_{2} \mathrm{O}\right), 6.43(\mathrm{~d}, 1 \mathrm{H}, J=16.5 \mathrm{~Hz}, \mathrm{ArCH}=\mathrm{CH})$, 6.80-7.10 (m, 3H, ArH), $7.52(\mathrm{~d}, 1 \mathrm{H}, J=16.5 \mathrm{~Hz}$, $\mathrm{ArCH}=\mathrm{CH})$.

\subsection{Synthesis of (E)-4-methoxymethyl-3-methoxycinnamic acid (5)}

To the compound $4(0.94 \mathrm{~g}, 3.7 \mathrm{mmol})$ in ethanol $(20 \mathrm{~mL}), 20 \mathrm{~mL}$ aqueous solution of $\mathrm{NaOH}(0.18 \mathrm{~g}$, $4.5 \mathrm{mmol}$ ) were added, then the mixture was heated under reflux for $3 \mathrm{~h}$. After that the reaction mixture was cooled and poured in acetic acid, which has been cooled 
in an ice bath. The solid product obtained was filtered and recrystallised (petroleum/acetic ether $=1 / 15$ ) to give the compound $\mathbf{5}$ ( $0.84 \mathrm{~g})$.

2.4a (E)-4-methoxymethyl-3-methoxycinnamic acid (5): This compound was obtained as a white solid, mp: $135-137^{\circ} \mathrm{C}$; $95 \%$ yield; HRMS calcd for $\mathrm{C}_{12} \mathrm{H}_{14} \mathrm{O}_{5}$ 238.0841, found 238.0848; ${ }^{1} \mathrm{H}$ NMR $(500 \mathrm{MHz}$, $\left.\mathrm{CDCl}_{3}\right) \quad \delta 3.52\left(\mathrm{~s}, 3 \mathrm{H}, \mathrm{OCH}_{3}\right), 3.92\left(\mathrm{~s}, 3 \mathrm{H}, \mathrm{OCH}_{3}\right)$, $5.28\left(\mathrm{~s}, 2 \mathrm{H}, \mathrm{OCH}_{2} \mathrm{O}\right), 6.33(\mathrm{~d}, 1 \mathrm{H}, J=16.0 \mathrm{~Hz}$, $\mathrm{ArCH}=\mathrm{CH}), 7.10-7.28(\mathrm{~m}, 3 \mathrm{H}, \mathrm{ArH}), 7.71(\mathrm{~d}, 1 \mathrm{H}, J=$ $16.0 \mathrm{~Hz}, \mathrm{ArCH}=\mathrm{CH})$.

\subsection{Synthesis of methyl (E)-3-[2-(4-hydroxy-3-} methoxyphenyl)-7-methoxy-3-methoxycarbonyl-2, 3-dihydro-1-benzofuran-5-yl]-prop-2-enoate $(\mathbf{6})^{15-17}$

Fresh silver oxide ( $1.56 \mathrm{~g}, 6.7 \mathrm{mmol})$ was added to a solution of compound $\mathbf{3}(2.81 \mathrm{~g}, 13.5 \mathrm{mmol})$ in dry acetone $(20 \mathrm{~mL})$ and toluene $(30 \mathrm{~mL})$ under a nitrogen atmosphere at $-20^{\circ} \mathrm{C}$. After stirring for $30 \mathrm{~h}$, the mixture was filtered and evaporated under reduced pressure. The residue was purified by a short silica gel column chromatography (petroleum/acetic ether $=3 / 1$ ) to give the compound 6 (1.27 g).

2.5a Methyl (E)-3-[2-(4-hydroxy-3-methoxyphenyl)-7methoxy-3-methoxycarbonyl-2,3-dihydro-1- benzofuran5-yl]-prop-2-enoate (6): This compound was obtained as a white crystals, mp: $151-152{ }^{\circ} \mathrm{C} ; 45 \%$ yield; HRMS calcd for $\mathrm{C}_{22} \mathrm{H}_{22} \mathrm{O}_{8} 414.1315$, found 414.1328; ${ }^{1} \mathrm{H}$ NMR $\left(500 \mathrm{MHz}, \mathrm{CDCl}_{3}\right) \quad \delta 3.74$ and $3.82(\mathrm{~s}, 6 \mathrm{H}, 2$ $\left.\times \mathrm{OCH}_{3}\right), 3.83$ and $3.94\left(\mathrm{~s}, 6 \mathrm{H}, 2 \times \mathrm{OCH}_{3}\right), 4.49(\mathrm{~d}$, $1 \mathrm{H}, J=8.0 \mathrm{~Hz}, \mathrm{H}-8), 6.03$ (d, $1 \mathrm{H}, J=8.0 \mathrm{~Hz}, \mathrm{H}-7$ ), $6.46\left(\mathrm{~d}, 1 \mathrm{H}, J=16.0 \mathrm{~Hz}, \mathrm{H}-8^{\prime}\right), 6.85-7.34(\mathrm{~m}, 5 \mathrm{H}$, ArH), 7.64 (d, 1H, $\left.J=16.0 \mathrm{~Hz}, \mathrm{H}-7^{\prime}\right) 7.69$ (s, 1H, OH). ${ }^{13} \mathrm{C}$ NMR $\left(125 \mathrm{MHz}, \mathrm{CDCl}_{3}\right) \quad \delta 51.67,53.07,55.92$, $56.35,56.54,87.54,110.97,113.46,116.03,116.29$, $119.02,120.19,127.36,129.42,131.81,145.50$, 145.80, 148.21, 148.75, 151.01, $167.86(\mathrm{C}=\mathrm{O}), 171.73$ $(\mathrm{C}=\mathrm{O})$. The data are consistant with the literature. ${ }^{12}$

2.6 Synthesis of methyl (E)-3-\{2-[4-(tetrahydro- $2 \mathrm{H}$ pyran-2-yloxy)-3-methoxyphenyl]-7-methoxy-3methoxycarbonyl-2,3-dihydro-1-benzofuran-5-yl\}prop-2-enoate $(7)^{18,19}$

A $25 \mathrm{~mL}$ dried round-bottom flask containing anhydrous dichloromethane $5 \mathrm{~mL}$ was charged with compound 6 (0.83 g, $2.0 \mathrm{mmol})$, PPTS $(0.05 \mathrm{~g}, 0.2 \mathrm{mmol})$ and DHP (0.21 g $2.5 \mathrm{mmol})$. After the mixture was stirred for $4 \mathrm{~h}$ at room temperature, the reaction completed. The residue was chromatographed on a silica gel column (petroleum/acetic ether $=4 / 1$ ) to give the compound $7(0.85 \mathrm{~g})$.

2.6a Methyl (E)-3-\{2-[4-(tetrahydro-2H-pyran-2yloxy)-3-methoxyphenyl]-7- methoxy-3-methoxycarbonyl-2, 3-dihydro-1-benzofuran-5-yl\}-prop-2-enoate (7): This compound was obtained as a pale yellow oil, $85 \%$ yield; HRMS calcd for $\mathrm{C}_{27} \mathrm{H}_{30} \mathrm{O}_{9} 498.1889$, found 498.1995; ${ }^{1} \mathrm{H}$ NMR $\left(500 \mathrm{MHz}, \mathrm{CDCl}_{3}\right) \quad \delta$ 1.59-1.94 (m, 6H, $\left.\mathrm{CH}_{2} \mathrm{CH}_{2} \mathrm{CH}_{2}\right), 3.56-3.73\left(\mathrm{~m}, 2 \mathrm{H}, \mathrm{OCH}_{2}\right), 3.72$ and $3.84\left(\mathrm{~s}, 6 \mathrm{H}, 2 \times \mathrm{OCH}_{3}\right), 3.88$ and $3.92(\mathrm{~s}, 6 \mathrm{H}, 2 \times$ $\left.\mathrm{OCH}_{3}\right), 4.35$ (d, $\left.1 \mathrm{H}, J=8.0 \mathrm{~Hz}, \mathrm{H}-8\right), 5.39(\mathrm{t}, 1 \mathrm{H}$, OCHO), 6.12 (d, 1H, $J=8.0 \mathrm{~Hz}, \mathrm{H}-7), 6.30$ (d, 1H, $J=$ $\left.16 \mathrm{~Hz}, \mathrm{H}-8^{\prime}\right), 6.77-7.19$ (m, 5H, ArH), $7.63(\mathrm{~d}, 1 \mathrm{H}, J=$ $\left.16 \mathrm{~Hz}, \mathrm{H}-7^{\prime}\right) .{ }^{13} \mathrm{C}$ NMR $\left(125 \mathrm{MHz}, \mathrm{CDCl}_{3}\right) \quad \delta 18.79$, $25.23,30.28,51.65,52.90,55.45,55.62,56.16,62.12$, 87.33, 97.02, 110.37, 112.18, 115.57, 117.80, 117.97, $118.80,125.74,128.64,133.65,144.45,144.78$, $146.63,150.02,150.52,167.63(\mathrm{C}=\mathrm{O}), 170.77(\mathrm{C}=\mathrm{O})$.

\subsection{Synthesis of 3-\{2-[4-(Tetrahydro-2H-pyran-2-} yloxy)-3-methoxyphenyl]-3-hydroxymethyl-7-methoxy2,3-dihydro-1-benzofuran-5-yl\}propan-1-ol (8) ${ }^{12,20}$

In a $50 \mathrm{~mL}$ dried three-necked flask, $\mathrm{LiAlH}_{4}(0.19 \mathrm{~g}$, $5.0 \mathrm{mmol}$ ) was dissolved in $30 \mathrm{~mL}$ of dry THF, and compound 7 (0.50 g, $1.0 \mathrm{mmol})$ was added slowly. The mixture was stirred at $0^{\circ} \mathrm{C}$ under a nitrogen atmosphere for $3 \mathrm{~h} . \mathrm{H}_{2} \mathrm{O}(0.19 \mathrm{~mL})$ was added, followed by treatment with $\mathrm{HCl}$, and the mixture was extracted with AcOEt $(3 \times 10 \mathrm{~mL})$. Then the organic phase was washed with saturated $\mathrm{NaCl}$ solution $(20 \mathrm{~mL})$, dried over $\mathrm{MgSO}_{4}$ and evaporated. The residue was chromatographed on a silica gel column (petroleum/acetic ether $=1 / 1)$ to give the compound $\mathbf{8}(0.34 \mathrm{~g})$.

2.7a 3-\{2-[4-( Tetrahydro-2H-pyran - 2 - yloxy ) - 3 methoxyphenyl]-3-hydroxymethyl-7-methoxy-2,3-dihydro1-benzofuran-5-yllpropan-1-ol (8): This compound was obtained as a yellow oil, 77\% yield; HRMS calcd for $\mathrm{C}_{25} \mathrm{H}_{32} \mathrm{O}_{7} 444.2148$, found $444.2156 ;{ }^{1} \mathrm{H}$ NMR $\left(500 \mathrm{MHz}, \mathrm{CDCl}_{3}\right) \quad \delta 1.60-1.96(\mathrm{~m}, 6 \mathrm{H}$, $\mathrm{CH}_{2} \mathrm{CH}_{2} \mathrm{CH}_{2}$ ), 1.86-2.08 (m, 2H, H-8'), $2.56(\mathrm{t}, 2 \mathrm{H}$, H-7'), 3.50-3.53 (m, 2H, $\left.\mathrm{OCH}_{2}\right), 3.55-3.56(\mathrm{~d}, 1 \mathrm{H}, J=$ $7.0 \mathrm{~Hz}, \mathrm{H}-8), 3.70$ (t, 2H, H-9'), 3.81 (s, 3H, $\mathrm{OCH}_{3}$ ), $3.82\left(\mathrm{~s}, 3 \mathrm{H}, \mathrm{OCH}_{3}\right), 4.03-4.09(\mathrm{~m}, 2 \mathrm{H}, \mathrm{H}-9), 5.30$ (t, 1H, OCHO), $5.47(\mathrm{~d}, 1 \mathrm{H}, J=7.0 \mathrm{~Hz}, \mathrm{H}-7), 6.59-7.01$ (m, 5H, ArH). $\left.{ }^{13} \mathrm{C} \mathrm{NMR} \mathrm{(125} \mathrm{MHz,} \mathrm{CDCl}_{3}\right) \quad \delta: 18.85$, 
$25.22,30.28,31.91,34.48,53.85,56.01,56.12,60.87$, $62.36,63.91,87.60,97.60,109.92,110.38,116.39$, $118.03,118.62,128.20,135.40,135.84,143.97$, $145.28,145.92,150.27$.

2.8 Synthesis of 2-[4-(tetrahydro-2H-pyran-2-yloxy)3-methoxyphenyl]-5-[3-(4-methoxymethyl-3-

methoxycinnamoyloxy)propyl]-3-[(4-methoxymethyl-3methoxycinnamoyloxy)propyl]-

7-methoxybenzodihydrofuran $(\mathbf{9})^{21}$

Under a nitrogen atmosphere, a $50 \mathrm{~mL}$, oven dried, round-bottom flask containing anhydrous dichloromethane $(20 \mathrm{~mL})$ was charged with acid $5(0.119 \mathrm{~g}$, $0.5 \mathrm{mmol})$, compound $8(0.111 \mathrm{~g}, 0.25 \mathrm{mmol})$, dicyclohexyl carbodiimide (DCC, $0.105 \mathrm{~g}, 0.5 \mathrm{mmol}$ ), and 4dimethylaminopyridine (DMAP, $0.021 \mathrm{~g}, 0.165 \mathrm{mmol}$ ) at $0^{\circ} \mathrm{C}$. The ice bath was removed after the addition was completed, and the resulting solution was stirred for $6 \mathrm{~h}$ at room temperature. The reaction mixture was filtrated and the solvent was distilled off. The residue was purified by flash column chromatography to afford compound 9 (0.103 g).

2.8a 2 - [ 4 - ( Tetrahydro - 2 H - pyran - 2-yloxy )-3methoxyphenyl]-5-[3-(4-methoxymethyl-3-methoxcinnamoyloxy) propyl]-3-[(4-methoxymethyl-3-methoxcinnamoyloxy) propyl]-l-7-methoxybenzodihydrofuran (9): This compound was obtained as a yellow oil, $47 \%$ yield; HRMS calcd for $\mathrm{C}_{49} \mathrm{H}_{56} \mathrm{O}_{15} 884.3619$, found 884.3628; ${ }^{1} \mathrm{H}$ NMR $\left(500 \mathrm{MHz}, \mathrm{CDCl}_{3}\right) \quad \delta$ 1.54-2.17 (m, 6H, $\mathrm{CH}_{2} \mathrm{CH}_{2} \mathrm{CH}_{2}$ ), 1.92-2.04 (m, 2H, H-2'"), 2.72 (t, 2H, $\left.\mathrm{H}-1^{\prime \prime \prime}\right), 3.49$ (s, $\left.3 \mathrm{H}, \mathrm{OCH}_{3}\right), 3.51$ (s, 3H, $\left.\mathrm{OCH}_{3}\right), 3.60$ $3.64\left(\mathrm{~m}, 2 \mathrm{H}, \mathrm{OCH}_{2}\right), 3.75(\mathrm{~m}, 1 \mathrm{H}, \mathrm{H}-3), 3.85$ (s, $3 \mathrm{H}$, $\left.\mathrm{OCH}_{3}\right), 3.91\left(\mathrm{~s}, 3 \mathrm{H}, \mathrm{OCH}_{3}\right), 3.92\left(\mathrm{~s}, 3 \mathrm{H}, \mathrm{OCH}_{3}\right), 3.93$ $\left(\mathrm{s}, 3 \mathrm{H}, \mathrm{OCH}_{3}\right), 4.05(\mathrm{dd}, 1 \mathrm{H}, J=7.0$ and $12.0 \mathrm{~Hz}$, $\left.\mathrm{H}-1^{\prime \prime}\right), 4.43\left(\mathrm{dd}, 1 \mathrm{H}, J=7.0\right.$ and $\left.12.0 \mathrm{~Hz}, \mathrm{H}-1^{\prime \prime}\right)$, $4.84\left(\mathrm{t}, 2 \mathrm{H}, J=6.5 \mathrm{~Hz}, \mathrm{H}-3^{\prime \prime \prime}\right), 5.07$ (s, 1H, OCHO), $5.27\left(\mathrm{~s}, 4 \mathrm{H}, 2 \times \mathrm{OCH}_{2} \mathrm{O}\right), 5.60(\mathrm{~d}, 1 \mathrm{H}, J=6.5 \mathrm{~Hz}$, H-2), 6.27 (d, 1H, $J=16.0 \mathrm{~Hz}, \mathrm{H}-\mathrm{a}), 6.35$ (d, 1H, $J=$ $\left.16.0 \mathrm{~Hz}, \mathrm{H}-\mathrm{a}^{\prime}\right), 6.67-7.16(\mathrm{~m}, 11 \mathrm{H}, \mathrm{ArH}), 7.50(\mathrm{~d}, 1 \mathrm{H}$, $J=16.0 \mathrm{~Hz}, \mathrm{H}-\mathrm{b}), 7.61\left(\mathrm{~d}, 1 \mathrm{H}, J=16.0 \mathrm{~Hz}, \mathrm{H}-\mathrm{b}^{\prime}\right)$. ${ }^{13} \mathrm{C}$ NMR $\left(125 \mathrm{MHz}, \mathrm{CDCl}_{3}\right) \quad \delta 19.19,24.96,25.21$, $30.27,33.91,50.51,55.94\left(2 \times \mathrm{OCH}_{3}\right), 56.16(2 \times$ $\left.\mathrm{OCH}_{3}\right), 56.34\left(2 \times \mathrm{OCH}_{3}\right), 62.12,63.78,65.27,88.72$, $95.14,97.50,104.20,110.25,110.45,110.86,115.60$, $115.64,115.91,116.23,116.24,117.75,118.85$, $121.36,122.28,122.51,127.76,128.49,128.86$, $134.44,134.75,144.19,144.82,145.33,145.53$, 146.37, 148.22, 148.51, 148.74, 149.74, 149.88, 166.89 $(\mathrm{C}=\mathrm{O}), 167.01(\mathrm{C}=\mathrm{O})$.
2.9 Synthesis of 2-(4-hydroxy-3-methoxyphenyl)-5-[3(4-hydroxy-3-methoxycinnamoyloxy) propyl]-3-(4hydroxy-3-methoxycinnamoyloxmethyl)-7methoxybenzodihydrofuran (Boehmenan)

In a $25 \mathrm{~mL}$ round-bottom flask, a mixture of $9(0.097 \mathrm{~g}$, $0.11 \mathrm{mmol})$ and PPTS (2.52 g, $0.011 \mathrm{mmol})$ in anhydrous ethanol $(8 \mathrm{ml})$ were stirred for $2 \mathrm{~h}$ at $55^{\circ} \mathrm{C}$. The solvent was removed in vacuo to give a crude product. To the residue of $25 \mathrm{ml}$ (round-bottomed flask), hydrochloric acid $(4 \mathrm{ml})$ in THF solution was added. Then the mixture was stirred for $0.5 \mathrm{~h}$ at room temperature and it was concentrated in vacuum. The crude product was purified by flash chromatography to afford Boehmenan (59.05 mg).

2.9a 2-(4-Hydroxy-3-methoxyphenyl)-5-[3-(4-hydroxy3 - methoxcinnamoyloxy)propyl] - 3 - hydroxymethyl - 7methoxybenzodihydrofuran (Boehmenan): This compound was obtained as yellow oil, 75\% yield; HRMS calcd for $\mathrm{C}_{40} \mathrm{H}_{40} \mathrm{O}_{12}$ 712.2520, found 712.2536; $1 \mathrm{H}$ NMR $\left(500 \mathrm{MHz}, \mathrm{CDCl}_{3}\right) \delta 1.97-2.06(\mathrm{~m}, 2 \mathrm{H}$, $\left.\mathrm{H}-2^{\prime \prime \prime}\right), 2.71\left(\mathrm{t}, 2 \mathrm{H}, J=7.6 \mathrm{~Hz}, \mathrm{H}-1^{\prime \prime \prime}\right), 3.83$ (s, 3H, $\left.\mathrm{OCH}_{3}\right), 3.90\left(\mathrm{~s}, 3 \mathrm{H}, \mathrm{OCH}_{3}\right), 3.93\left(\mathrm{~s}, 6 \mathrm{H}, 2 \times \mathrm{OCH}_{3}\right)$, $3.84-3.93$ (m, 1H, H-3), 4.23 (t, $2 \mathrm{H}, J=6.5 \mathrm{~Hz}, \mathrm{H}-$ $\left.3^{\prime \prime \prime}\right), 4.43\left(\mathrm{dd}, 1 \mathrm{H}, J=7.7\right.$ and $\left.11.2 \mathrm{~Hz}, \mathrm{H}-1^{\prime \prime}\right), 4.59$ (dd, $1 \mathrm{H}, J=5.1$ and $\left.11.2 \mathrm{~Hz}, \mathrm{H}-1^{\prime \prime}\right), 5.50(\mathrm{~d}, 1 \mathrm{H}, J$ = $7.8 \mathrm{~Hz}, \mathrm{H}-2), 5.65(\mathrm{~s}, 1 \mathrm{H}, \mathrm{OH}), 5.91(\mathrm{~s}, 1 \mathrm{H}, \mathrm{OH})$, $5.92(\mathrm{~s}, 1 \mathrm{H}, \mathrm{OH}), 6.23(\mathrm{~d}, 1 \mathrm{H}, J=15.9 \mathrm{~Hz}, \mathrm{H}-\mathrm{a})$, $6.30\left(\mathrm{~d}, 1 \mathrm{H}, J=15.9 \mathrm{~Hz}, \mathrm{H}-\mathrm{a}^{\prime}\right), 6.69-7.09(\mathrm{~m}, 11 \mathrm{H}$, ArH), 7.49 (d, 1H, $J=15.9 \mathrm{~Hz}, \mathrm{H}-\mathrm{b}), 7.61(\mathrm{~d}, 1 \mathrm{H}, J=$ $\left.15.9 \mathrm{~Hz}, \mathrm{H}-\mathrm{b}^{\prime}\right) .{ }^{13} \mathrm{C} \mathrm{NMR}\left(125 \mathrm{MHz}, \mathrm{CDCl}_{3}\right) \quad \delta 30.71$, $32.13,50.67,55.90\left(2 \times \mathrm{OCH}_{3}\right), 56.01\left(2 \times \mathrm{OCH}_{3}\right)$, $63.68,65.42,88.89,108.76,109.32,109.39,112.38$, $114.21,114.71,114.72,114.73,115.37,116.12$, $119.71,122.97,123.14,126.67,126.94,127.43$, $132.50,134.89,144.08,144.92,145.48,145.66$, $146.23,146.64,146.71,146.73,147.94,148.12,167.02$ $(\mathrm{C}=\mathrm{O}), 167.34(\mathrm{C}=\mathrm{O})$. The data are consistant with the literature. ${ }^{1}$

\subsection{The formation of the dihydrobenzofuran neolignan (10-12)}

2.10a The formation of methyl (E,E)-4,4'-dihydroxy3,5'-dimethopxy- $\beta$-3'-bicinnamate (10): The compound 6 ( $82.9 \mathrm{mg}, 0.2 \mathrm{mmol})$ was dissolved in acetone $(10 \mathrm{ml})$ with $\mathrm{KOH}(22.4 \mathrm{mg}, 0.4 \mathrm{mmol})$ and stirred at room temperature for $4 \mathrm{~h}$. The solution was acidified with $\mathrm{HCl}$ and partitioned between EtOAc and saturated $\mathrm{NaCl}$. The organic layer was dried over $\mathrm{MgSO}_{4}$, and 
then crystallized (petroleum/acetic ether $=2 / 1$ ) to give compound 10 (62.1 mg).

2.10b Methyl (E,E)-4,4'-dihydroxy-3,5'-dimethopxy$\beta$-3'-bicinnamate (10): This compound was obtained as pale yellow solid, $75 \%$ yield; mp. $148-149{ }^{\circ} \mathrm{C}$; HRMS calcd for $\mathrm{C}_{22} \mathrm{H}_{22} \mathrm{O}_{8} 414.1315$, found 414.1326; ${ }^{1} \mathrm{H}$ NMR (500 MHz, $\left.\mathrm{CDCl}_{3}\right) \quad \delta 3.50\left(\mathrm{~s}, 3 \mathrm{H}, \mathrm{OCH}_{3}\right)$, $3.78\left(\mathrm{~s}, 3 \mathrm{H}, \mathrm{OCH}_{3}\right), 3.79\left(\mathrm{~s}, 3 \mathrm{H}, \mathrm{OCH}_{3}\right), 3.94(\mathrm{~s}$, $\left.3 \mathrm{H}, \mathrm{OCH}_{3}\right), 5.74(\mathrm{~s}, 1 \mathrm{H}, \mathrm{OH}), 6.00(\mathrm{~s}, 1 \mathrm{H}, \mathrm{OH}), 6.26$ $\left(\mathrm{d}, 1 \mathrm{H}, J=16.0 \mathrm{~Hz}, \mathrm{H}-8^{\prime}\right), 6.57-7.07$ (m, 5H, Ar-H), $7.56\left(\mathrm{~d}, 1 \mathrm{H}, J=16.0 \mathrm{~Hz}, \mathrm{H}-7^{\prime}\right), 7.83$ (s, 1H, H-7). ${ }^{13} \mathrm{C}$ NMR $\left(125 \mathrm{MHz}, \mathrm{CDCl}_{3}\right) \quad \delta$ 52.02, 52.06, 55.22, $56.62,110.59,113.47,115.58,115.73,124.53,125.19$, $125.63,125.66,126.13,126.22,140.68,145.07$, 147.58, 147.66, 148.72, 148.78, $167.02(\mathrm{C}=\mathrm{O}), 167.40$ $(\mathrm{C}=\mathrm{O})$.

2.10c The formation of (E,E)-4,4'-dihydroxy-3,5'dimethopxy- $\beta$-3'-bicinnamic acid (11): The compound $6(82.9 \mathrm{mg}, 0.2 \mathrm{mmol})$ was dissolved in $10 \%$ $\mathrm{NaOH}(10 \mathrm{ml})$ and stirred at room temperature for $4 \mathrm{~h}$. The mixture was acidified with $\mathrm{HCl}$ and partitioned between EtOAc and saturated $\mathrm{NaCl}$. The organic layer was dried over $\mathrm{MgSO}_{4}$, and then crystallized (petroleum/acetic ether $=2 / 1$ ) to give compound $\mathbf{1 1}$ (70.3 mg).

2.10d (E,E)-4,4'-dihydroxy-3,5'-dimethopxy- $\beta$-3'-bicinnamic acid (11): This compound was obtained as pale yellow oil, 91\% yield; $\mathrm{HRMS}$ calcd for $\mathrm{C}_{20} \mathrm{H}_{18} \mathrm{O}_{8}$ 386.1002, found 386.1013; ${ }^{1} \mathrm{H}$ NMR $(500 \mathrm{MHz}$, $\left.\mathrm{CDCl}_{3}\right) \quad \delta 3.88\left(\mathrm{~s}, 3 \mathrm{H}, \mathrm{OCH}_{3}\right), 3.92\left(\mathrm{~s}, 3 \mathrm{H}, \mathrm{OCH}_{3}\right)$, $6.37\left(\mathrm{~d}, 1 \mathrm{H}, J=16.0 \mathrm{~Hz}, \mathrm{H}-8^{\prime}\right), 6.71-7.37(\mathrm{~m}, 5 \mathrm{H}$, Ar-H), $7.60\left(\mathrm{~d}, 1 \mathrm{H}, J=16.0 \mathrm{~Hz}, \mathrm{H}-7^{\prime}\right), 7.82(\mathrm{~s}, 1 \mathrm{H}$, $\mathrm{H}-7), 12.15$ (s, 2H, $2 \times \mathrm{COOH}) .{ }^{13} \mathrm{C}$ NMR $(125 \mathrm{MHz}$, $\left.\mathrm{CDCl}_{3}\right) \quad \delta$ 55.93, 56.45, 110.27, 113.27, 116.24, $125.14,125.62,126.34,126.37,127.26,127.59$, $141.81,145.81,147.85,148.02,148.96,149.12$, $155.64,168.56(\mathrm{C}=\mathrm{O}), 169.18(\mathrm{C}=\mathrm{O})$. The data are consistant with the literature. ${ }^{22}$

2.10e The formation of (E)-4-hydroxy-3-(2-[(E)-4hydroxy-3-methoxystyryl]-15-methoxycinnamic acid (12): The compound 6 (82.9 mg, $0.2 \mathrm{mmol})$ was dissolved in $10 \% \mathrm{NaOH}(10 \mathrm{ml})$ and stirred for $4 \mathrm{~h}$ under reflux. Then the reaction solution was cooled and concentrated. The mixture was acidified with $\mathrm{HCl}$ and partitioned between EtOAc and saturated $\mathrm{NaCl}$. The organic layer was dried over $\mathrm{MgSO}_{4}$, and then crystallized (petroleum/acetic ether $=2 / 3$ ) to give compound $12(36.9 \mathrm{mg})$.

2.10f (E)-4-hydroxy -3-(2- $[(E)-4$ - hydroxy -3methoxystyryl]-35-methoxycinnamic acid (12): This compound was obtained as pale yellow oil, 54\% yield; HRMS calcd for $\mathrm{C}_{19} \mathrm{H}_{18} \mathrm{O}_{6} 342.1103$, found 342.1109; ${ }^{1} \mathrm{H}$ NMR $\left(500 \mathrm{MHz}, \mathrm{CDCl}_{3}\right) \quad \delta 3.74\left(\mathrm{~s}, 3 \mathrm{H}, \mathrm{OCH}_{3}\right)$, $3.83\left(\mathrm{~s}, 3 \mathrm{H}, \mathrm{OCH}_{3}\right), 6.46\left(\mathrm{~d}, 1 \mathrm{H}, J=15.5 \mathrm{~Hz}, \mathrm{H}-8^{\prime}\right)$, 6.76-7.52 (m, 5H, Ar-H), 7.21(d, 1H, J = 15.5 Hz, H-7), $7.22(\mathrm{~d}, 1 \mathrm{H}, J=15.5 \mathrm{~Hz}, \mathrm{H}-8), 7.53(\mathrm{~d}, 1 \mathrm{H}, J=15.5 \mathrm{~Hz}$, H-7'), 9.14 (s, 1H, Ph-OH), 9.40 (s, 1H, Ph-OH), 12.20 $(\mathrm{s}, 1 \mathrm{H}, \mathrm{COOH}) \cdot{ }^{13} \mathrm{C}$ NMR $\left(125 \mathrm{MHz}, \mathrm{CDCl}_{3}\right) \quad \delta$ 56.06, 56.56, 109.55, 110.26, 116.17, 116.70, 119.95, $120.09,120.37,125.09,125.90,129.60,129.89$, $145.19,146.37,147.11,148.32,148.58,168.50(\mathrm{C}=\mathrm{O})$. The data are consistant with the literature. ${ }^{22}$

\section{Results and discussion}

The analytic and spectroscopic data of Boehmenan and the intermediate products are given in experimental section.

Our approach to the synthesis of natural product Boehmenan 1 is outlined in scheme 2. Vanillin was used as starting material. Compound $\mathbf{3}$ was formed through Knoevenagel condensation between vanillin and methyl hydrogen malonate. The 4-hydroxyl group of $\mathbf{3}$ was protected with $\mathrm{MOMCl}$ to afford the product $\mathbf{4}$, which was hydrolysed to form compound $\mathbf{5}$.

The preparation of compound $\mathbf{6}$ from ferulic acid methyl ester 3 by biomimetic oxidative coupling with $\mathrm{Ag}_{2} \mathrm{O}$ to construct the skeleton of dihydrobenzofuran lignan was the key step in our method. The reaction gave different yield dependent on the reaction conditions. As shown in table 1, freshly prepared $\mathrm{Ag}_{2} \mathrm{O}$ was used in the reaction into compound $\mathbf{7}$ with higher yield than that of conventional $\mathrm{Ag}_{2} \mathrm{O}$ and recycling $\mathrm{Ag}_{2} \mathrm{O}$. The solvent system had only small effect on the yield of 7, but the reaction temperature and time had significant influence in the reaction. A decrease in the reaction temperature increased the yield of compound $\mathbf{6}$, while the by product was decreased. Moreover, in the process of the reaction, the yield increased to a maximum and began to decrease as the time increased further. The optimum reaction conditions were obtained as follows: freshly prepared $\mathrm{Ag}_{2} \mathrm{O}$, dry acetone $(20 \mathrm{~mL})$ and toluene $(30 \mathrm{~mL}), 30 \mathrm{~h}$ and $-20{ }^{\circ} \mathrm{C}$. Under above conditions, the yield of compound 6 was $45 \%{ }^{23,24}$ 


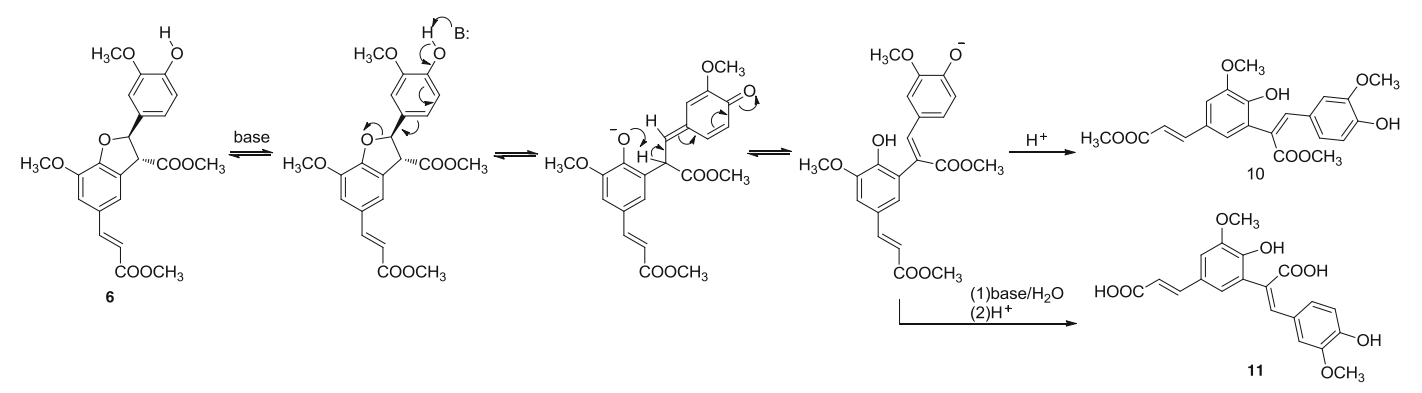

Scheme 2. Formation of the stilbene $\mathbf{1 0}$ and diacid $\mathbf{1 1}$ from diester $\mathbf{6}$.

Conversion of $\mathbf{6}$ into intermediate $\mathbf{8}$ was carried out by two steps: protection of dimers $\mathbf{6}$ with THP group and reduction of the resulting compound 7 with $\mathrm{LiAlH}_{4}$.

The protection of hydroxyl group as MOM ether is a commonly used transformation in synthetic organic chemistry. So the methoxymethyl chloride was firstly chosen as a protecting reagent for free hydroxyl in the presence of dry $\mathrm{K}_{2} \mathrm{CO}_{3}$ and acetone, but no reaction occurred. When treated with $\mathrm{KOH}$ in THF, compound $\mathbf{6}$ was transformed to ring-opening product 10. Finally, the target products 7 was gained by using DHP with PPTS as protecting reagent. Thus, to research the ringopenning reaction of compound $\mathbf{6}$ in the presence of acid and base, various conditions, such as certain base, solvent, temperature and time, were tested.

The results from table 2 show that the ring-openning compound $\mathbf{1 0}$ was obtained in the condition of entry 16. When strongly basic with $\mathrm{H}_{2} \mathrm{O}$ was used as solvent, the diacid $\mathbf{1 1}$ was formed at room temperature with $91 \%$ yield (entry 11), while at reflux, the monoacid $\mathbf{1 2}$ was gained (entry 12). Treatment of the diester $\mathbf{6}$ with weak base $\mathrm{K}_{2} \mathrm{CO}_{3}$ in the conditions (entry 7-9) did not produce any product, but at reflux in $\mathrm{H}_{2} \mathrm{O}$, the compound 6 was transformed into $\mathbf{1 1}$ with $83 \%$ yield (entry 10).

The formation of $\mathbf{1 0}$ and $\mathbf{1 1}$ is proposed by the mechanism presented in scheme 2. At basic conditions, the attack of the nucleophile at the hydroxy-H seems to initiate the opening of the dihydrobenzofuran ring to a quinone methide intermediate, subsequent elimination of H-proton and restoration of the aromatic ring system, followed by the formation of the compound $\mathbf{1 0}$ after acidification at room tempreture in organic solvent. But in $\mathrm{H}_{2} \mathrm{O}$, the saponified products $\mathbf{1 1}$ is obtained.

The mechanism for the formation of compound $\mathbf{1 2}$ is shown in scheme 3 . In aqueous sodium hydroxide solution under heating, the hydrolysis of diester $\mathbf{6}$ results in the cyclic diacid. After the decarboxylation, the acyclic products $\mathbf{1 2}$ is obtained with the openning of the dihydrobenzofuran ring.

The intermediate 8 was condensated with compound 5 in the presence of DCC and DMAP at room temperature to form compound $\mathbf{9}$. The removal of the protecting groups using PPTS and $\mathrm{HCl}$ at room temperature afforded the target product Boehmenan.

\section{Conclusion}

In summary, we have developed an efficient synthesis strategy of Boehmenan which was based on biomimetic oxidative coupling to construct the skeleton of lignan. The synthetic method has the advantages of

Table 1. Comparison of different reaction conditions and oxidant effect.

\begin{tabular}{lllccc}
\hline Entry & Preparation of $\mathrm{Ag}_{2} \mathrm{O}$ & Solvent & T $\left({ }^{\circ} \mathrm{C}\right)$ & Time $(\mathrm{h})$ & Yield (\%) \\
\hline 1 & Freshly prepared $\mathrm{Ag}_{2} \mathrm{O}$ & Acetone/toluene & 25 & 8 & 21 \\
2 & Freshly prepared $\mathrm{Ag}_{2} \mathrm{O}$ & Acetone/toluene & 25 & 24 & 41 \\
3 & Freshly prepared $\mathrm{Ag}_{2} \mathrm{O}$ & Acetone/toluene & 25 & 32 & 33 \\
4 & Recycling $\mathrm{Ag}_{2} \mathrm{O}$ & Acetone/toluene & 25 & 24 & 17 \\
5 & Conventional $\mathrm{Ag}_{2} \mathrm{O}$ & Acetone/toluene & 25 & 24 & 21 \\
6 & Freshly prepared $\mathrm{Ag}_{2} \mathrm{O}$ & $\mathrm{CH}_{2} \mathrm{Cl}_{2}$ & 25 & 24 & 43 \\
7 & Freshly prepared $\mathrm{Ag}_{2} \mathrm{O}$ & Acetone/toluene & 0 & 28 & 39 \\
8 & Freshly prepared $\mathrm{Ag}_{2} \mathrm{O}$ & Acetone/toluene & 0 & 34 & 45 \\
9 & Freshly prepared $\mathrm{Ag}_{2} \mathrm{O}$ & Acetone/toluene & -25 & 30 & 38 \\
10 & Freshly prepared $\mathrm{Ag}_{2} \mathrm{O}$ & Acetone/toluene & -25 & 36 & \\
\hline
\end{tabular}


Table 2. The ring-opening reaction of compound $\mathbf{6}$ under different conditions.

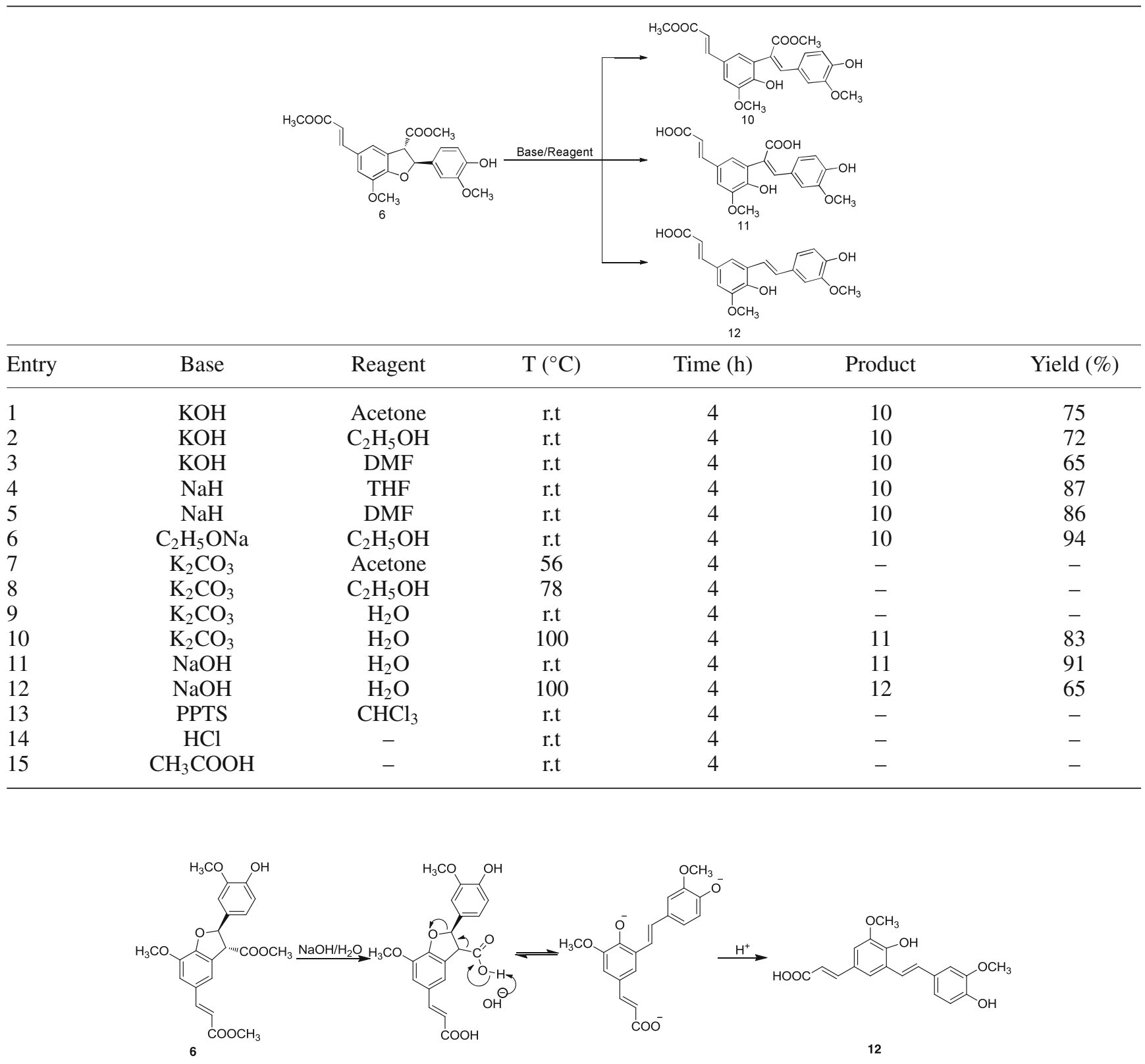

Scheme 3. Formation of the compound $\mathbf{1 2}$ from compound 6.

easy availability of starting materials and simple operation. So it has considerable practical value. By this route, natural compound Boehmenan was synthesized in 8 steps, with an yield of $8 \%$ for the first time. In addition, the ring-openning reaction of dihydrobenzofuran neolignan intermediate was discussed in this paper.

\section{Acknowledgements}

This work was supported by the National Natural Science Foundation of Shandong (No. ZR2010HM023), the Chinese Medicine Administration Bureau of Gansu Province (No.GZK-2011-62).

\section{References}

1. Seca A M L, Silva A M S, Silvestre A J D, Cavaleiro J A S, Domingues F M J and Pascoal-Neto C 2001 Phytochemistry $\mathbf{5 6} 759$

2. Wu P L, Wu T S, He C X, Su C H and Lee K H 2005 Chem. Pharm. Bull. 5356

3. Rudiyansyah T and Garson M J 2006 J. Nat. Prod. 69 1218

4. Chin Y M, Jones W P, Rachman I, Riswan S, Kardono L B S, Chai H B, Farnsworth N R, Cordell G A, Swanson S M, Cassady J M and Kinghorn A D 2006 Phytother. Res. 2062

5. Sasaki T, Li W, Morimura H, Li S, Li Q, Asada Y and Koike K 2011 Chem. Pharm. Bull. 591396

6. Ward R S 1995 Nat. Prod. Rep. 12183 
7. Koubaa I and Damak M 2003 Fitoterapia 7418

8. Yang X W, Zhao P J, Ma Y L, Xiao H T, Zuo Y Q, He H P, Li L and Hao X J 2007 J. Nat. Prod. 70521

9. Chen J J, Yang C S, Peng C F, Chen I S and Miaw C L 2008 J. Nat. Prod. 711016

10. Cutillo F, D’Abrosca B, DellaGreca M, Fiorentino A and Zarrelli A 2003 J. Agric. Food Chem. 516165

11. Xia Y M, Wang W, Yang F K and Chang L 2010 Chem. Res. Chin. Univ. 31947

12. Pieters L, Dyck S V, Gao M, Bai R, Hamel E, Vlietinck A and Lemiere G 1999 J. Med. Chem. 425475

13. Ihara M, Suzuki M, Fukumoto K, Kametani $\mathrm{T}$ and Kabuto C 1988 J. Am. Chem. Soc. 1101963

14. Kumar P, Raju S V N, Reddy R S and Pandey B 1994 Tetrahedron Lett. 351289

15. Orlandi M, Rindone B, Molteni G, Rummakko P and Brunow G 2001 Tetrahedron 57371
16. Apers S, Paper D, Burgermeister J, Baronikova S, Dyck S V, Lemiere G, Vlietinck A and Pieters L 2002 J. Nat. Prod. 65728

17. Zhou L X and Lin M 2000 Chin. Chem. Lett. 11515

18. Patney H K 1991 Synth. Commun. 212329

19. Hon Y S, Lee C F, Chen R J and Szu P H 2001 Tetrahedron $\mathbf{5 7} 5991$

20. Eklund P C, Riska A and Sjoholm R E 2002 J. Org. Chem. 677544

21. Bringmaann G, Breuning M and Henschel P 2004 Org. Synth. 10448

22. Ralph J, Quideau S, Grabber J H and HatfieId R D 1994 Chem. Soc. Perkin Trans. 13485

23. Bruschi M, Orlandi M, Rindone B, Rummakko P and Zoia L 2006 J. Phys. Org. Chem. 19592

24. Sako M, Hosokawa H, Ito T and Iinuma M $2004 \mathrm{~J}$. Org. Chem. 692598 